\title{
On Channel-Adaptive Fair Multiple Access Control
}

\author{
Li Wang, Yu-Kwong Kwok, Wing-Cheong Lau, and Vincent K. N. Lau \\ Department of Electrical and Electronic Engineering \\ The University of Hong Kong, Pokfulam Road, Hong Kong
}

\begin{abstract}
Multiple access control (MAC) of the uplink in a wireless mobile computing system is one of the most important resource allocation problems in that the response time and throughput of user applications (e.g., wireless Web surfing) are critically affected by the efficiency of the MAC protocol. Compared with a traditional MAC problem (e.g., the wireline Ethernet), there are two important new challenges in a modern wireless network: (1) multimedia data with diverse traffic requirements are involved; and (2) the wireless channel has a time-varying quality for each user. Furthermore, a more prominent user requirement is fairness among different users, possibly with different traffic demands. While some protocols have been suggested to handle multimedia data and/or tackling the time-varying channel, there are a number of drawbacks in these existing protocols. The most notable drawback is that the channel model is rather unrealistic-just using a two state Markov chain instead of relying on accurate models of multipath fading and shadowing effects. Another common deficiency is that fairness is ignored. In this paper, we propose to use a new notion of fairness that can capture a realistic channel model, and to integrate a fair queueing scheduling algorithm in a MAC protocol to optimize performance while maintaing fairness among users regardless of their channel states and data types.
\end{abstract}

Keywords: fairness, multiple access control, scheduling, channel-adaptive, wireless multimedia, mobile computing.

\section{INTRODUCTION}

The MAC problem has been receiving significant attention from the research community and a myraid of techniques have been proposed for various different classical and practical formulations [3], [17], [18]. In recent years, as multimedia wireless communications become important, many useful MAC protocols have also been suggested to efficiently handle the heterogeneous nature of the traffic characteristics [8]. Lately, it is widely envisioned that the time-varying nature of the wireless channel quality [15], [19], inevitable as it is, can potentially allow further exploitation to enhance the performance of the system [4], [22]. As such, several channel-adaptive protocols have also been reported [2]. However, for the MAC problem, existing channel-adaptive protocols just blindly maximize the overall system throughput by always selecting the user device with the best channel quality and allowing such a user to seize the channel to transmit data [2], [8]. A typical approach is to sort the users in descending order of channel quality and then assign transmission time-slots to the one user after another until all the time-slots in the current multiple access frame are exhausted [8]. The drawback of using such an approach is that the fairness [21] among the users is completely ignored. Thus, while users with good channel conditions can enjoy a higher throughput and shorter delay, users with poor channel conditions may be unable to satisfy his/her traffic demands (e.g., continuously dropping video or voice packets).

In this paper, we focus on the fairness aspect in a channeladaptive MAC protocol. Specifically, we consider a fair queueing scheduler, called CAFQ (Channel-Adaptive Fair Queueing) [20], which intelligently uses the channel state informa- tion (CSI), to be incorporated in the MAC protocol to guarantee fairness among users while maximizing system utilization. An overview of the CAFQ algorithm is provided in Section III. As indicated by the results shown in Section IV, the proposed CAFQ based MAC for uplink access, can indeed provide better performance in terms of delay, throughput, and packet loss for a wide range of system parameters. Before introducing CAFQ approach, in Section II we describe the system models used in our study. The final section of this paper provides some concluding remarks.

\section{System Models}

The mobile computing system considered in this paper, with a star topology (i.e., a cellular phone network with file and media data transmission support), is aimed to support integrated video, voice, and file data services (hereafter we refer file data as simply data).

\section{A. Source Models}

Video and voice packets are assumed to be delay sensitive while data packets are assumed to be delay insensitive. Thus, voice packets are labeled with deadlines. A voice packet will be dropped by the mobile device if the deadline expires before being transmitted. Such packet dropping has to be controlled to within a certain limit (e.g., below $1 \%$ as indicated in [6]) in order that the quality of service to the voice users is still acceptable. The source and contention models are summarized below.

- Voice Source Model: The voice source is assumed to be continuously toggling between the talkspurt and silence states. The duration of a talkspurt and a silence period are assumed to be exponentially distributed with means $t_{t}$ and $t_{s}$ seconds, respectively (as indicated by the empirical study in [6], $t_{t}=1$, and $\left.t_{s}=1.35\right)$. We assume a talkspurt and a silence period start only at a frame boundary.

- Video Source Model: We use video teleconferencing as an example video source. In the model we use [10], the number of packets per video frame period (i.e., $40 \mathrm{msec}$ for a $25 \mathrm{fps}$ frame rate) is govern by the DAR(1) model, which is a Markov chain characterized by three parameters: the mean, the variance, and $\rho$. The transition matrix is computed as:

$$
P=\rho I+(1-\rho) Q
$$

where $\rho$ is the autocorrelation coefficient and I is the identity matrix. Furthermore, each row of $Q$ is identical and consists of the negative binomial probabilities $\left(f_{0}, \ldots, f_{K}, F_{K}\right)$, where $F_{K}=\sum_{k<K} f_{k}$, and $K$ is the peak rate. Similar to a voice source, a video source can only tolerate a $1 \%$ packet loss rate [10]. 
- Data Source Model: The arrival time of file data generated by a mobile device is assumed to be exponentially distributed with mean equal to one second. The data size, in terms of number of packets, is also assumed to be exponentially distributed with mean equal to 100 packets. Again we assume that the packets arrive at a frame boundary.

- Request Contention Model: As in most previous studies, to avoid excessive collisions, even if a voice or data request has some packets awaiting to be sent, the mobile device will attempt to send a request at a request mini-slot (described in detailed below) only with a certain permission probability. The permission probability for submitting voice and data requests are denoted by $p_{v}$ and $p_{d}$, respectively.

A mobile device entering a new voice talkspurt or generating a new stream of data packets transmits an appropriate request packet in one of the request slots of the next frame. If there are more than one packet transmitted in the same request slot, collision occurs and none of the requests will be correctly received (we ignore the capture effect [1] in this paper). At the end of each request slot, the successful or unsuccessful request will be identified and broadcast by the base-station. An unsuccessful mobile device (does not receive the acknowledgment announcement in the downlink frame) can retry in the next request slot. On the other hand, a successful mobile device then transmits his/her information packet in the corresponding information slot in the current frame.

\section{B. Channel and Transmission Models}

The wireless link between a mobile device and the basestation is characterized by two components, namely the fast fading component and the long-term shadowing component. Fast fading is caused by the superposition of multipath components and is therefore fluctuating in a very fast manner (on the order of a few msec). Long-term shadowing is caused by terrain configuration or obstacles and is fluctuating only in a relatively much slower manner (on the order of one to two seconds).

Redundancy is incorporated to the information packet for error protection. To exploit the time-varying nature of the wireless channel, a variable-throughput channel-adaptive physical layer is employed [9]. CSI (channel state information), $c(t)$, which is estimated at the receiver, is fed back to the transmitter via a lowcapacity feedback channel. Based on the CSI, the level of redundancy and the modulation constellation applied to the information packets are adjusted accordingly by choosing a suitable transmission mode. Thus, the instantaneous throughput is varied according to the instantaneous channel state. In our study, a 6mode variable-throughput adaptive bit-interleaved trellis coded modulation scheme (ABICM) is employed [9]. Transmission modes with normalized throughput ${ }^{1}$ varying from $1 / 2$ to $5 / 6$ are available depending on the channel condition. The essence of incorporating a channel-adaptive physical layer such as the $\mathrm{ABICM}$ in the MAC protocol is to capture the notion of multiple channel quality levels such that the scheduler in the MAC protocol can exploit the scheduling diversity to optimize overall system performance. Note that using ABICM is just for illustration only and certainly other channel-adaptive transmission

\footnotetext{
${ }^{1}$ Normalized throughput refers to the number of information bits carried per modulation symbol.
}

models/techniques [22] can be used.

We assume the coherence time of the short-term fading is around ten msec which is much longer than an information slot duration (which is $2.5 \mathrm{msec}$ ). Thus, the CSI remains approximately constant within at least two frames and it follows that the transmission mode for the whole frame is determined only by the current CSI level. Most importantly, scheduling decisions made at the beginning of a frame are based on accurate channel condition information about the frame yet to be transmitted. Specifically, transmission mode $q$ is chosen if the feedback CSI, $\hat{c}$, falls within the adaptation thresholds, $\left(\zeta_{q-1}, \zeta_{q}\right)$. Here, the operation and the performance of the ABICM scheme is determined by the set of adaptation thresholds $\left\{\zeta_{0}, \zeta_{1}, \ldots\right\}$. In this paper, we assume that the ABICM scheme is operated in the constant BER mode [9]. That is, the adaptation thresholds are set optimally to maintain a target transmission error level over a range of CSI values. When the channel condition is good, a higher mode could be used and the system enjoys a higher throughput. On the other hand, when the channel condition is bad, a lower mode is used to maintain the target error level at the expense of a lower transmission throughput. Note that when the channel state is very bad, the adaptation range of the ABICM scheme can be exceeded such that the throughput (mode-0) becomes so low, making it impossible to maintain the targeted BER level.

Given the above considerations about the channel state, the instantaneous throughput offered to the access control layer, denoted by $\rho$, is also variable and is therefore a function of the CSI, $c(t)$, and the target BER, $P_{b}$, denoted by $\rho=f_{\rho}\left(c(t), P_{b}\right)$.

\section{Channel-Adaptive FaIR Queueing MAC}

\section{A. Fairness Notions for Wireless Networks}

In our study, we have considered the following existing scheduling algorithms for wireless networks: WPS (Wireless Packet Scheduling) [12], IWFQ (Idealized Wireless Fair Queueing Algorithm) [12], CIF-Q (Channel-Condition Independent Fair Queueing) [14], SBFA (Server Based Fairness Algorithm) [16], CS-WFQ (Channel State Independent Wireless Fair Queueing) [11], ELF (Effort Limited Fairness) [5], Proportional Fairness [7], and WFS (Wireless Fair Service) [13].

On the surface, these previous algorithms work well in that they schedule the error-free sessions to transmit data while leaving the error sessions (in a bad channel state) waiting until their channel states become good again. Thus, to maintain fairness, it suffices to guarantee that the error sessions can catch up (i.e., get back the missing service share) within a bounded period of time. However, usually nothing can be said about the behavior and the time bound of the error period. Furthermore, the key assumption, which, we believe, is the major drawback, is that a session in a bad channel state can transmit nothing. This is undeniably an over-simplification in view of the fact that channel-adaptive and variable rate physical layer protocols are commonly sought to combat the time-varying nature of wireless channels. Algorithms that use such a simplified assumption include: CIF-Q, IWFQ, SBFA, and WFQ. On the other hand, the more practical algorithms, such as the ELF, CS-WFQ (uses a similar principle as in ELF), proportional fair, and the CAFQ algorithm, allow 
sessions to transmit packets even though the sessions are in a non-perfect channel state (hence, effort is very likely not equal to outcome).

\section{B. Overview of the CAFQ Algorithm}

The CAFQ algorithm [20] has the following distinctive features:

- a new notion of fairness is employed;

- contrary to CIF-Q, graceful degradation is not ensured to help the lagging session more efficiently;

- a punish factor is used to decide how seriously the scheduler punishes a non-perfect channel state session that transmit packets; and

- a virtual compensation session is incorporated to help the lagging sessions to catch up.

We believe that, from the user's viewpoint, fairness should be maintained in that so long as a session can transmit some data, it should be provided with some chance to transmit. At the same time, QoS should also be met. However, from the system manager's viewpoint, its hard to meet these two sometimes conflicting goals with a limited bandwidth and channels that have time-varying quality. Because whenever a session without a perfect channel state is allowed to transmit, there will be part of the bandwidth wasted, and the wasted bandwidth can never be replenished. It should be noted that this is very different from the idea of swapping sessions that are error-free and error-prone, as in existing scheduling algorithms such as CIF-Q. When an errorfree session takes the opportunity of an error-prone sessions, it will relinquish the service when the error-prone one is in a good channel state.

Indeed, if abundant bandwidth is available or the channel state is most likely to be perfect, we should maintain the graceful degradation, and prevent the leading sessions from starving. But in a realistic system in which the channel is usually not so good, we cannot expect to achieve perfect allocations, but rather we should meet the sessions QoS first. Thus, in the CAFQ algorithm, graceful degradation is not implemented and the rationale is to compensate the lagging sessions as soon as possible so as to quickly resume a higher throughput and to reduce the delay.

\section{Channel-Adaptive Fairness}

We use a notion of fairness to be maintained in the short term, called channel-adaptive fairness (CAF). Specifically, a scheduler is channel-adaptive fair if in the short term the difference between the normalized throughput (normalized with respect to the channel capacity) of any two backlogged sessions $i$ and $j$ is bounded as follows:

$$
\left|\frac{T_{i}\left(t_{1}, t_{2}\right)}{r_{i} f\left(\Phi_{i}\right)}-\frac{T_{j}\left(t_{1}, t_{2}\right)}{r_{j} f\left(\Phi_{j}\right)}\right|<\epsilon
$$

where $\Phi_{i}$ denotes the channel state (e.g., one of the five classes $\mathrm{A}, \mathrm{B}, \mathrm{C}, \mathrm{D}$, and $\mathrm{E})$, and $f\left(\Phi_{i}\right)=M\left(\Phi_{i}\right)^{\eta}$ in which $M\left(\Phi_{i}\right)$ is the effective throughput factor $\left(0 \leq M\left(\Phi_{i}\right) \leq 1\right)$. The effective throughput factor is channel state dependent: $M\left(\Phi_{i}\right)=0.75$ if $\Phi_{i}$ is channel state $\mathrm{B}$, and so on. Here, $\eta$ is a punish factor which is a positive number. Thus, in our definition of fairness, the throughput a session receives will be proportional to its channel quality. And, in the long term, outcome fair is maintained among all sessions.

The channel-adaptive fairness is more reasonable in the wireless environment because it considers explicitly the different channel states. Unlike the CIF-Q algorithm that prevents the sessions without perfect channel state from transmitting and unlike the ELF algorithm that distributes the normalized amount of service inversely proportional to their channel states, a CAF scheduler provides transmission opportunities to all sessions that do not suffer from the worst channel state in the short term, and at the same time, it punishes the sessions without good channel states to different extent. Furthermore, unlike the proportional fair scheduler, using the CAF scheduler does not necessarily schedule the session with the best channel condition to transmit first. With the channel-adaptive fairness, we can formalize a new fair queueing algorithm, which is explicated in detailed in the following section.

The punish factor $\eta$ can help to decide between to make use of the bandwidth more efficiently and to treat every session more fairly. When a larger value of punish factor is used, we punish the non-perfect channel state session that transmit packets more seriously, and prevent it from wasting too much bandwidth. In effect, the bandwidth is used more efficiently, and the average delay of the total system is decreased and the throughput is increased. But if there is a session that is more unlucky than the others and have a higher probability of having a bad channel state, its average delay and throughput may be very bad, because it is punished seriously and prevented from occupying the bandwidth. When a smaller punish factor is used, this kind of unlucky sessions will be punished only moderately, so the average delay and throughput of these sessions are improved. But as they have more chance to access the bandwidth and hence incur a larger wastage of bandwidth, the total throughput and average delay of the system will be adversely affected. Thus, there is a trade-off and the punish factor can be used to tune the utilization of system resources.

\section{RESUlts}

In this section, we present the detailed results of our performance comparison of the three protocols (CIF-Q, PF, and CAFQ) applied to the multiple access control problem with system model as described in Section II (i.e., an accurate channel model and the ABICM scheme are used). Mobile users are assumed to be moving in a random way at a maximum speed of $40 \mathrm{~km} / \mathrm{hr}$ in a microcell environment. Table I summarizes the parameters we used.

In the simulations, we tested the algorithms with and without an request queue, which stores at the base-station the previous requests that survive the contention but are not allocated information slots. Such a request queue can further alleviate the capacity loss due to requests contention, especially when the traffic load is high. However, including a request queue inevitably increases the implementation complexity and space requirements of the algorithms in a practical situation. Thus, there is a tradeoff.

Figure 1 shows the no-queue results for the following test scenarios: 
TABLE I

SIMULATION PARAMETERS.

\begin{tabular}{lr}
\hline Parameter & Value \\
\hline$N_{r}$ & 10 \\
$N_{i}$ & 40 \\
$N_{b}$ & 20 \\
$t_{t}$ & $1000 \mathrm{msec}$ \\
$t_{s}$ & $1350 \mathrm{msec}$ \\
$p_{c}$ & 0.3 \\
$p_{v}$ & 0.3 \\
$p_{a}$ & 0.2 \\
punish factor $(\eta)$ & 2.5 \\
channel bandwidth & $1.36 \mathrm{MHz}$ \\
voice data rate & $8 \mathrm{kbps}$ \\
video data rate & $128 \mathrm{kpbs}$ \\
file data rate & $16 \mathrm{kbps}$ \\
number of simulated frames & $2 \times 10^{6}$ \\
\hline
\end{tabular}

- video data loss rate for 5-50 video service users (with 25 data service users and 30 voice service users);

- voice data loss rate for 10-240 voice service users (with 25 data service users and 20 video service users);

- data service delay and throughput for 5-50 data service users (with 25 video users and 30 voice users).

Each data point is an average of 50 trials of the same configuration with different random seeds. As can be seen, the CAFQ algorithm outperforms the CIF-Q algorithm significantly in all of the four performance metrics: data throughput, data delay, voice packet loss rate, and video packet loss rate. Most importantly, the CAFQ algorithm also outperforms the PF algorithm considerably. This is very encouraging because PF is widely considered to be an effective fair scheduling technique and has been adopted in CDMA HDR services implementation.

Figure 2 shows the results with a request queue added to all algorithms. Interestingly, adding a request queue improves the performance of all the algorithms. A plausible explanation is that with a request queue, the number of requests available for scheduling (selection) is much larger, and thus, such scheduling diversity allows the scheduler to make more accurate decisions while maintaining the fairness among requests. Similar to the no-queue cases, the CAFQ performs the best among the three algorithms.

\section{CONCLUSIONS}

Multiple access control (MAC) of the uplink in a startopology based mobile computing system is a very important problem that demands a high quality solution such that system utilization is maximized while satisfying users' requests which include fairness. Because of the time-varying nature of the wireless channel, various channel-adaptive techniques have been proposed previously to exploit the error properties in order to further enhance system utilization. However, fairness is usually ignored in these techniques. In this paper, by using a new notion of fairness which can capture a very realistic model of the wireless channel, we propose to use a channel-adaptive fair queueing approach, called CAFQ, in the MAC protocol. The

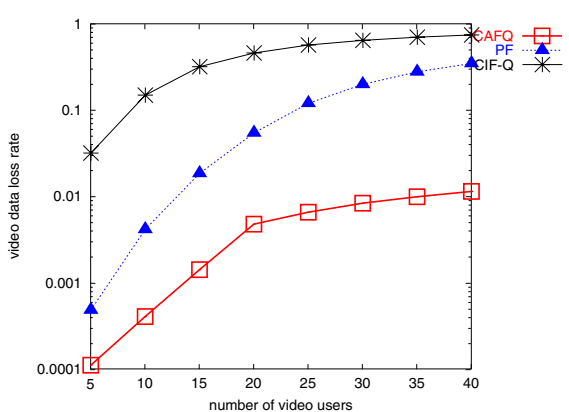

(a) video packet loss rate

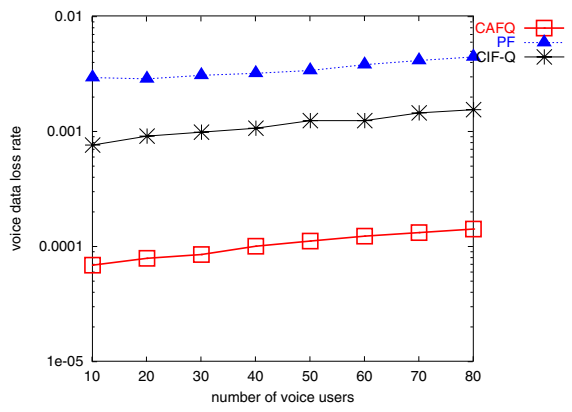

(b) voice packet loss rate

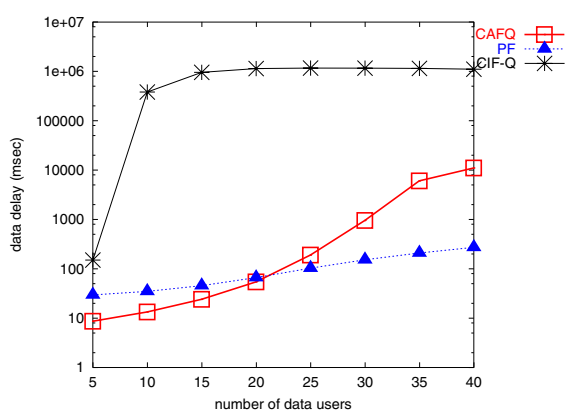

(c) data service delay

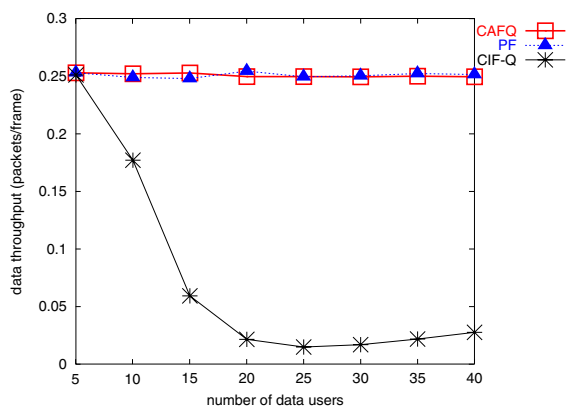

(d) data service throughput

Fig. 1. Performance of the three algorithms without using a request queue. 


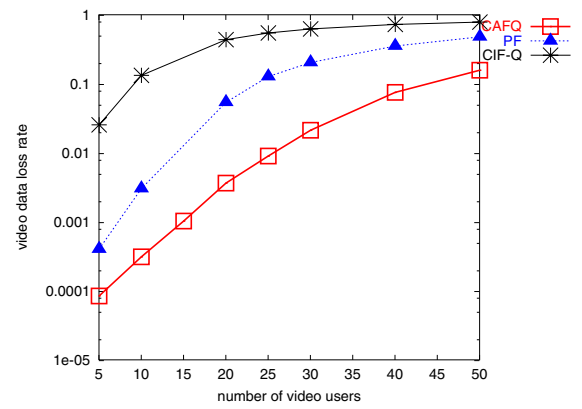

(a) video packet loss rate

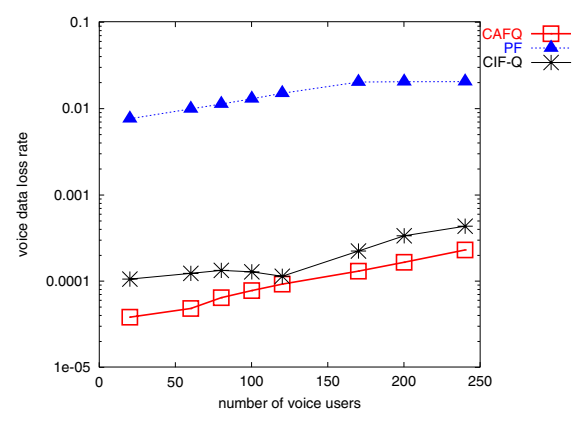

(b) voice packet loss rate

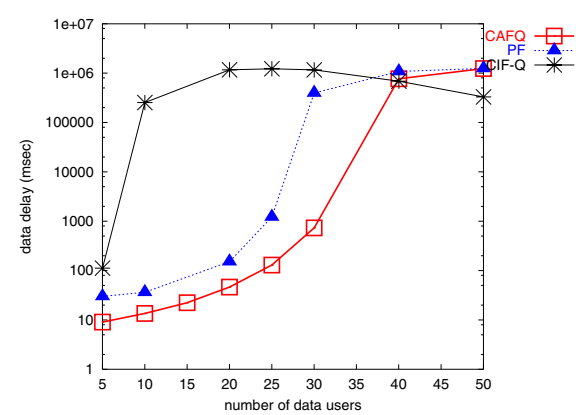

(c) data service delay

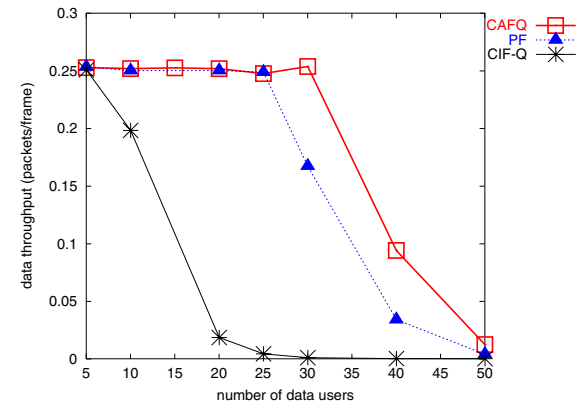

(d) data service throughput
CAFQ-based MAC is found to have better MAC performance compared to several existing techniques including CIF-Q and proportional fair queueing.

\section{ACKNOWLEDGMENTS}

This research was supported by the Hong Kong Research Grants Council under project number HKU 7024/00E.

\section{REFERENCES}

[1] J. C. Ambak and W. van Blitterswijk, "Capacity of Slotted ALOHA in Raleigh Fading Channels," IEEE Journal on Selected Areas in Communications, vol. SAC-5, no. 2, pp. 261-268, Feb. 1987.

[2] P. Bhagwat, P. Bhattacharya, A. Krishna, and S. K. Tripathi, "Using Channel State Dependent Packet Scheduling to Improve TCP Throughput over Wireless LANs," ACM/Baltzer Wireless Networks, vol. 3, no. 1, pp. 91$102,1997$.

[3] A. Brand and H. Aghvami, Multiple Access Protocols for Mobile Communications, John Wiley \& Sons, 2002.

[4] A. Chockalingam, M. Zorzi, and V. Tralli, "Wireless TCP Performance with Link Layer FEC/ARG,” Proceedings of ICC'99, pp. 1212-1216, June 1999.

[5] D. A. Eckhardt and P. Steenkiste, "Effort-Limited Fair (ELF) Scheduling for Wireless Networks," Proc. INFOCOM'2000, pp. 1097-1106, 2000.

[6] J. Gruber and L. Strawczynski, "Subjective Effects of Variable Delay and Speech Clipping in Dynamically Managed Voice Systems," IEEE Transactions on Communications, vol. COM-33, vol. 8, pp. 801-808, Aug. 1985.

[7] A. Jalali, R. Padovani, and R. Pankaj, "Data Throughput of CDMA-HDR: A High Efficiency High Data Rate Personal Communication Wireless System," Proc. VTC'2000.

[8] Y.-K. Kwok and V. K. N. Lau, "A Quantitative Comparison of Multiple Access Control Protocols for Wireless ATM," IEEE Transactions on Vehicular Technology, vol. 50, no. 3, pp. 796-815, May 2001.

[9] V. K. N. Lau, "Performance of Variable Rate Bit-Interleaved Coding for High Bandwidth Efficiency,' Proc. of VTC'2000, vol. 3, pp. 2054-2058, May 2000.

[10] T. V. Lakshman, A. Ortega, and A. R. Reibman, "VBR Video: Tradeoffs and Potentials," Proceedings of the IEEE, vol. 86, no. 5, pp. 952-973, May 1998.

[11] P. Lin, B. Bensaou, Q. L. Ding, and K. C. Chua, "A Wireless Fair Scheduling Algorithm for Error-Prone Wireless Channels," Proc. WoWMoM'2000, pp. 11-20, 2000

[12] S. Lu, V. Bharghavan, and R. Srikant, "Fair Scheduling in Wireless Packet Networks," IEEE/ACM Trans. Networking, vol. 7, no. 4, pp. 473-489, Aug. 1999.

[13] T. Nandagopal, S. Lu, and V. Bharghavan, "A Unified Architecture for the Design and Evaluation of Wireless Fair Queueing Algorithms," Proc. MOBICOM'99, pp. 132-142, 1999.

[14] T. S. E. Ng, I. Stoica, and H. Zhang, "Packet Fair Queueing Algorithms for Wireless Networks with Location-Dependent Errors," Proc. INFOCOM'98, pp. 1103-1111, 1998.

[15] J. D. Parsons, The Mobile Radio Propagation Channel, Second Edition, Wiley, 2000.

[16] P. Ramanathan and P. Agrawal, "Adapting Packet Fair Queueing Algorithms to Wireless Networks," Proc. MOBICOM'98, pp. 1-9, 1998.

[17] R. Rom and M. Sidi, Multiple Access Protocols: Performance and Analysis, Springer Verlag, 1990.

[18] S. Tasaka, Performance Analysis of Multiple Access Protocols, MIT Press, 1986.

[19] D. N. C. Tse and S. V. Hanly, "Multiaccess Fading Channels-Part I: Polymatroid Structure, Optimal Resource Allocation and Throughput Capacities," IEEE Trans. Information Theory, vol. 44, no. 7, Nov. 1998, pp. 2796-2815.

[20] L. Wang, Y.-K. Kwok, W.-C. Lau, and V. K. N. Lau, "Channel Capacity Fair Queueing in Wireless Networks: Issues and A New Algorithm," Proceedings of the 2002 IEEE International Conference on Communications (ICC'2002), vol. 5, pp. 3116-3120, Apr. 2002.

[21] H. Zhang, "Service Disciplines for Guaranteed Performance Service in Packet-Switching Networks," Proceedings of the IEEE, vol. 83, no. 10, Oct. 1995, pp. 1374-1396.

[22] M. Zorzi and R. R. Rao, "The Role of Error Correlations in the Design of Protocols for Packet Switched Services," Proceedings of the 35th Annual Allerton Conference on Communications, Control, and Computing, pp. 749-758, Sept. 1997.

Fig. 2. Performance of the three algorithms with a request queue. 